\title{
Analysis of physical plasma properties within flare kernels from EUV/X-ray observations
}

\author{
S. Gburek, J. Sylwester, B. Sylwester, M. Kowalinski \\ Space Research Centre, Polish Academy of Sciences, Wroclaw ul. Kopernika 11, Poland \\ email: sg@cbk.pan.wroc.pl
}

\begin{abstract}
We performed the study of a solar flare of GOES class X1.0 which occurred on 2002 August 21, peak time 05:34 UT. A new method was applied for temperature determination of the plasma seen in TRACE images. This method makes use of the detailed knowledge of the TRACE point spread function (PSF) function. Substantial dependence of TRACE PSF on wavelength allows for determination of temperature from a single TRACE image even in these portions of the image which are saturated. We also determined the average temperature of this flare from the soft X-ray spectra measured by RESIK Bragg spectrometer aboard CORONAS-F satellite. The results obtained from TARCE and RESIK data are compared.
\end{abstract}

\section{Introduction}

We discuss properties of X1.0 solar flare which occurred on 21 August 2002 at S12W51 heliographic coordinates. The soft X-ray flux started to rise abruptly in GOES channels at 05:28 UT reaching peak emission at about 05:34 UT.

For this event there is particularly good coverage in spectroscopic data from RESIK instrument (Sylwester, J. et al. 2004) which are used here for determination of temperature and of the plasma in soft X-rays in various flare phases. The entire flare was also imaged in EUV by the TRACE telescope (Handy, B. N. et al. 1999). The TRACE data were recorded with 1 arcsec resolution and cadence of $17 \mathrm{~s}$. All images were taken in TRACE 195 band with exposures of $9.7 \mathrm{~s}$. In particular we selected for analysis TRACE frame recorded at 05:31:45 UT around the maximum of EUV flux when the strongest diffraction effects were present in the image.

We found that diffraction effects observed in TRACE images can be well characterized in terms of the telescope PSF. The TRACE PSF is a multi-peaked function. Except the main central peak (the core caused by geometrical imperfectness of the optics), the PSF contains also other peaks which are formed as replications of the core due to diffraction on the nickel wire mesh supporting the telescope entrance filters.

We constructed theoretical models of the TRACE PSF shape for 195 filter for a set of selected temperatures from the range 0.5 - 20.0 MK. In doing this we assumed that the emitting plasma is isothermal, concentrated in a point-like source. The solar spectra emitted were determined based on CHIANTI code (Dere et al. 2001). The shape of the central core peak was determined using the blind deconvolution algorithm.

The analysis of temperature dependence of calculated PSF models shows that due to small dispersion the low order maxima are almost exact copies of the central (zerothorder) structure shifted only by the appropriate distances. This suggests that, after background subtraction, signal values in the saturated image region can be reconstructed by shifting and rescaling the signal from first order maxima. On the other hand, far diffraction maxima shapes are very sensitive to temperature distribution in the central brightest region. Either their shapes or positions vary significantly for different temperatures. 

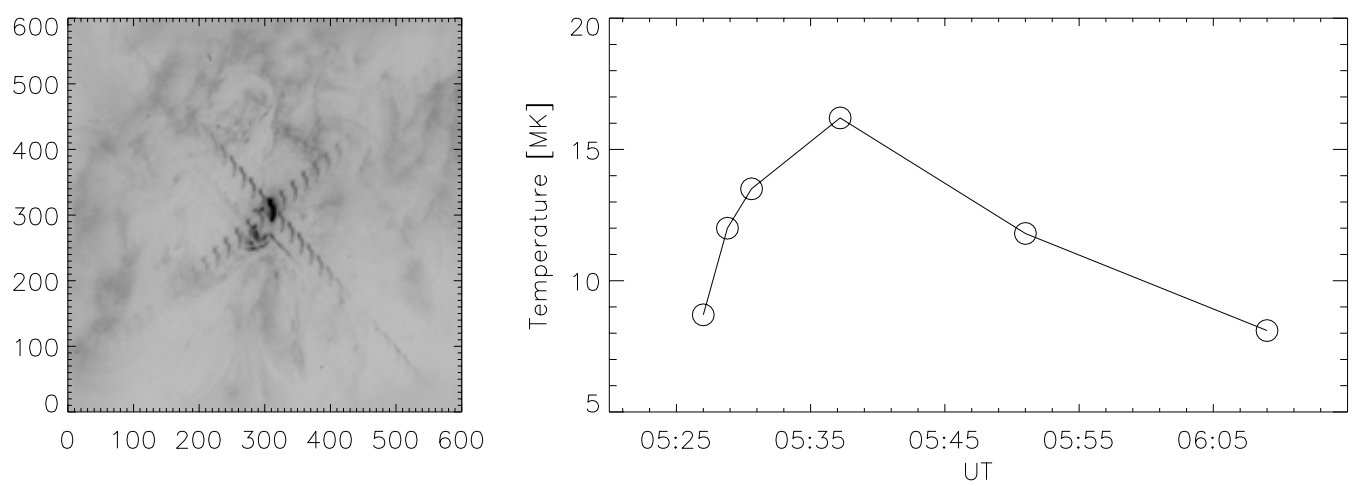

Figure 1. Left: TRACE 195 image of solar flare taken on 21 August 2002 at 05:31:45 UT.

Right: Evolution of the temperature determined from RESIK spectra for the entire event.

We performed the first attempt to temperature determination from the above mentioned TRACE image taken on 21 August 2002 at 05:31:45 UT. First we subtracted background and reconstructed the signal in saturated area using rescaled signal from first order diffraction maxima which were not affected by saturation. Next we redistributed the signal of the brightest flare kernel in the reconstructed image area using TRACE diffraction pattern properties determined from the PSF analysis. The redistribution was performed for a set of different temperatures in the range $0.5-20.0 \mathrm{MK}$ with step of $0.1 \mathrm{MK}$. In this way, for each temperature in the range, we obtained a theoretical model of the signal diffracted from the selected kernel. Eventually we checked for which temperature the theoretically modelled diffraction pattern best matches the real pattern observed in the data. We found that single temperature models gives rather poor quality fits to the data. Hence we decided to use simple two-temperature model for fitting. The model in which $90 \%$ of the kernel plasma is at temperature $2.3 \mathrm{MK}$ and $10 \%$ at $6.9 \mathrm{MK}$ provided a good quality of the fit.

We used RESIK soft X-ray spectra recorded close in time in order to determine the flare kernel temperature in the isothermal approximation. For this purpose we took the (lines + continuum) fluxes in the most energetically separated RESIK soft X-ray channels $3.30-$ $3.85 \AA$ and $4.85-6.00 \AA$. Determined RESIK value of the temperature is approximately twice higher $(T=13.5 \mathrm{MK})$ than the highest temperature obtained from TRACE image analysis what may indicate a multitemperature structure of the flaring kernel.

In conclusion we found that the diffraction pattern seen in TRACE data can be very useful in studies of the flaring plasma geometry and thermodynamic properties. Analysis of strong diffraction effects will allow for determination of plasma temperature, emission measure and their distributions even from a single image notwithstanding of severe central saturation which accompanies usually strong diffraction.

\section{Acknowledgements}

RESIK is a common project between NRL (USA), MSSL and RAL (UK), IZMIRAN (Russia) and SRC (Poland). The authors acknowledge support from grants 2.P03D.002.22 and PBZ-KBN-054/P03/2001 of the Polish Committee for Scientific Research.

\section{References}

Dere, K. P., Landi, E. Young, P. R. \& Del Zanna, G., 2001, ApJSS, 331-354.

Handy, B. N., Acton L. W. Kalkenborg C. C. \& 45 co-authors., 1999 Sol. Phys. 187, 229-260.

Sylwester, J., Gaicki I., Kordylewski Z. \& 19 co-authors., 2004, in preparation. 